TITLE:

\title{
Effects of side groups on the entanglement network of cellulosic polysaccharides
}

$\operatorname{AUTHOR}(S)$ :

Horinaka, Jun-ichi; Urabayashi, Yuhei; Takigawa, Toshikazu

CITATION:

Horinaka, Jun-ichi ...[et al]. Effects of side groups on the entanglement network of cellulosic polysaccharides. Cellulose 2015, 22(4): 2305-2310

ISSUE DATE:

2015-05-27

URL:

http://hdl.handle.net/2433/201993

\section{RIGHT:}

The final publication is available at Springer via http://dx.doi.org/10.1007/s10570-015-0670-7.; The full-text file will be made open to the public on 27 May 2016 in accordance with publisher's 'Terms and Conditions for Self-Archiving':; This is not the published version. Please cite only the published version.; この論文は出版社版でありません。引用の際には 出版社版をご確認ご利用ください。 
$9 *$ Corresponding author.

10 E-mail: horinaka.junichi.5c@kyoto-u.ac.jp

11 Tel: $+81-75-383-2454$

Fax: +81-75-383-2458 


\section{Abstract}

20 The transient entanglement network of cellulosic polysaccharides in concentrated solutions

21 were characterized by the molecular weight between entanglements $\left(M_{\mathrm{e}}\right)$ using dynamic

22 viscoelasticity measurements. From the concentration dependence of $M_{\mathrm{e}}, M_{\mathrm{e}}$ for the cellulosic

23 polysaccharides in the molten state $\left(M_{\mathrm{e}, \mathrm{melt}}\right)$ was estimated as the material constants reflecting

24 the chain characteristics. The values of $M_{\text {e,melt }}$ were compared among three cellulosic

25 polysaccharides: cellulose, methylcellulose, and hydroxypropyl cellulose. Methylcellulose and hydroxypropyl cellulose were employed as cellulose derivatives having small and large side groups, respectively. It appeared that hydroxypropyl cellulose had significantly larger $M_{\mathrm{e}, \mathrm{melt}}$ compared with cellulose and methyl cellulose. However, the numbers of repeating glucose-ring units between entanglements were very close to each other among the three polysaccharides.

34 Keywords entanglement network ' cellulosic polysaccharide ${ }^{\cdot}$ side group concentrated 


\section{Introduction}

40 Cellulose is the most familiar polysaccharide as the result of long years of use in human history.

41 In addition to cellulose in the natural form, many derivatives are known to be synthesized from

cellulose by modifying the hydroxyl groups. The main aim of introducing the substituents is to change the physical properties of cellulose; for example, methylcellulose is water-soluble in contrast to natural cellulose, because the methyl groups reduce the strength of hydrogen bonding between cellulose chains. Currently, cellulosic polysaccharides, meaning cellulose as well as its derivatives, are vital materials in several industries due to the various properties.

47 Cellulosic polysaccharides are also of interest in the field of chain characterization of polymers. The chain dimensions of cellulosic polysaccharides in various dilute solutions have been examined previously and the effects of side groups (substituents) on the unperturbed chain parameters as well as on the hydrodynamic properties have been discussed based on the accumulated data (Brown et al. 1963; Brown et al. 1964; Flory 1966; Kamide et al. 1978; Kamide et al. 1987). Thus chain characterization of cellulosic polysaccharides using dilute solutions has been successfully carried out. Studies on concentrated systems of cellulosic polysaccharides are also making good progress (Chen st al. 2009; Gericke et al. 2009; Haward et al. 2012; Kosan et al. 2008; Syang-Peng et al. 2009). A specific matter to concentrated polymer systems such as polymer melts and concentrated solutions is the entanglement coupling 
57 between polymer chains emerging from topological constraints of the interpenetrating polymer

chains. It is recognized that entanglement coupling dominates the rheological behavior of

concentrated polymer systems at long times (Doi et al. 1986; Ferry 1980). The entanglement corresponds to an average mesh size of the transient entanglement network. The value of $M_{\mathrm{e}}$ for characteristics obtained only by examining the rheological behavior of concentrated polymer systems. Until now, however, $M_{\mathrm{e}, \text { melt }}$ for the better part of cellulosic polysaccharides have been unknown and effects of side groups on entanglement coupling for cellulosic polysaccharides has not even been considered.

In this paper, $M_{\mathrm{e}, \text { melt }}$ for three cellulosic polysaccharides, cellulose (C), methylcellulose (MC), and hydroxypropyl cellulose (HPC), have been estimated from the rheological data for their concentrated solutions. It should be noted that MC has small substituents of methyl groups, while HPC has larger ones of hydroxypropyl groups, which is characterized by the molecular weight of a repeating unit $\left(M_{\text {unit }}\right)$ below. Use of an ionic liquid as a solvent has made it possible to prepare solutions of these cellulosic polysaccharides at high concentrations where there is entanglement coupling. The values of $M_{\mathrm{e}, \mathrm{melt}}$ as well as the number of repeating glucose-ring units between entanglements $\left(N_{\text {unit }}\right)$ have been compared among the three polysaccharides. 


\section{Experimental}

Materials

The cellulosic polysaccharides, C (Aldrich, USA), MC (Aldrich, USA), and HPC (Wako, Japan), were used without further purification. According to the manufacturers, the degree of substitution per repeating unit were 1.7 and 3.8 for MC and HPC, respectively; therefore, $M_{\text {unit }}$

81 for C, MC, and HPC were estimated to be 162, 186, and 383, respectively. The viscosities for $2 \%$ aqueous solutions of MC and $\mathrm{HPC}$ at $20{ }^{\circ} \mathrm{C}$ were reported by the manufacturers to be 4000 cP and 1000-4000 cP, respectively, whereas that for $\mathrm{C}$ was unavailable. An ionic liquid 1-butyl-3-methylimidazolium acetate (BmimAc; BASF, Germany) was used as received. As far as we tested, BmimAc was the only common solvent to prepare concentrated solutions of the three polysaccharides. Each polysaccharide sample was added into liquid BmimAc in a dry glass vessel, and then the mixture was stirred on a hot plate at about $80{ }^{\circ} \mathrm{C}$ for more than $6 \mathrm{~h}$ until complete dissolution. The concentration of the polysaccharides $(c)$ ranged from $1.1 \times 10^{2}$ to $2.1 \times 10^{2} \mathrm{kgm}^{-3}$ (ca. 10 to $20 \mathrm{wt} \%$ ) for $\mathrm{C}$, from $4.2 \times 10^{1}$ to $7.5 \times 10^{1} \mathrm{kgm}^{-3}$ (ca. 4 to $7 \mathrm{wt} \%$ )

90 for MC, from $5.2 \times 10^{1}$ to $2.6 \times 10^{2} \mathrm{kgm}^{-3}$ (ca. 5 to $25 \mathrm{wt} \%$ ) for HPC; the lowest $c$ was slightly

91 above the critical concentration for entanglement coupling, while the highest $c$ was determined

92 by the solubility. In the calculation of $c$, the densities of melts of the polysaccharides were 
93 commonly assumed to be $1.0 \times 10^{3} \mathrm{kgm}^{-3}$ and that of BmimAc was quoted to be $1.055 \times 10^{3}$

$94 \mathrm{kgm}^{-3}$ (Horinaka et al. 2013).

95

96 Rheological measurements

97 The angular frequency $(\omega)$ dependence of the storage modulus $\left(G^{\prime}\right)$ and the loss modulus $\left(G^{\prime \prime}\right)$

98 for the polysaccharide solutions was measured with an ARES rheometer (now TA Instruments,

99 USA) under a nitrogen atmosphere. The geometry for the measured sample was a cone-plate

100 with a diameter of $25 \mathrm{~mm}$ and a cone angle of $0.1 \mathrm{rad}$. The value of $\omega$ ranged from 0.1 to $100 \mathrm{~s}^{-1}$

101 and the amplitude of the oscillatory strain $(\gamma)$ was fixed at 0.1 so that the measurement could be

performed in the linear viscoelasticity region. The above measurement was carried out at several

\section{Results and Discussion}

Figure 1 shows the $\omega$ dependence of $G$ ' and $G$ " for the C solutions; the curves are shifted

curve at the reference temperature $\left(T_{\mathrm{r}}\right)$ of $80{ }^{\circ} \mathrm{C}$ obtained by means of a horizontal shift by a 
$111 \omega a_{\mathrm{T}}$, flow of the system can be seen for each $c$, although the terminal relation of $G " \propto \omega$ is

112 observed only for the solution of $c=1.1 \times 10^{2} \mathrm{kgm}^{-3}$. In the middle $\omega a_{T}$ region, there is a

113 plateau region in each $G^{\prime}$ curve where $G^{\prime}>G^{\prime}$, which becomes wider as $c$ increases; this is the

114 so-called rubbery plateau, indicating the existence of entanglements coupling in the C solutions.

115 The reason that the plateau of $G$ ' is slightly tilted is probably the polydispersity of the sample

116 employed, although actual data on the polydispersity are not available. In Figures $2, \log a_{\mathrm{T}}$ is

117 plotted against $1 / T$. All data points can be fitted by a single line regardless of $c$, as drawn in the

118 figure, indicating that the $T$-dependence curve of $a_{\mathrm{T}}$ can be represented by an Arrhenius-type

119 equation. This trend implies that the solutions are homogeneous.

120 Figure 3 shows the master curves of the $\omega$ dependence of $G$, and $G$ " at $T_{\mathrm{r}}=80^{\circ} \mathrm{C}$ for the MC

121 solutions obtained in the similar way to Figure 1. Although the rubbery plateau region in each

$122 G^{\prime}$ curve is not obvious compared with that in Figure 1 partly due to the low $c$, the rubbery

123 plateau as well as the flow region is seen for each $c$ in the $G$ ' and $G$ " curves, which is typical of

124 polymer solutions with entanglement coupling. The $T$-dependence curve of $a_{\mathrm{T}}$ for the MC

125 solutions is shown in Figure 4. The values of $a_{\mathrm{T}}$ at a given $T$ are almost identical regardless of $c$,

126 and all data points in the figure appear to fall on a single line.

127 The master curves of the $\omega$ dependence of $G^{\prime}$ and $G^{\prime \prime}$ at $T_{\mathrm{r}}=80{ }^{\circ} \mathrm{C}$ for the HPC solutions are

128 given in Figure 5. The rubbery plateau region becomes wider as $c$ increases, so that the flow 
129 region goes out of sight for the solution of $c=2.1 \times 10^{2} \mathrm{kgm}^{-3}$. The Arrhenius-type plot of $a_{\mathrm{T}}$

130 for the HPC solutions is shown in Figure 6. As is the case of $\mathrm{C}$ and $\mathrm{MC}$, the data points fall on a

131 single line; namely, $a_{\mathrm{T}}$ for these solutions show almost the same $T$-dependence being

132 independent of $c$. This result supports that the HPC solutions are homogeneous within the $c$

133 range examined.

134 As demonstrated above, the rubbery plateau appears for all the solutions examined. From the analogy with the rubber elasticity, $M_{\mathrm{e}}\left(\right.$ in $_{\mathrm{gmol}}{ }^{-1}$ ) for a polymer in solution at the concentration of $c$ can be given by

$$
M_{\mathrm{e}}=\frac{10^{3} c R T}{G_{\mathrm{N}}^{0}}
$$

138 Here, $G_{\mathrm{N}}^{0}$ is the plateau modulus, which corresponds to the height of the plateau, and $R$ is the gas constant (Doi et al. 1986; Ferry 1980). It should be noted that $G_{\mathrm{N}}^{0}$ is independent of the molecular weight of the polymer itself as long as the molecular weight is sufficiently greater than $2 M_{\mathrm{e}}$ and the plateau appears (Ferry 1980; Onogi 1970). As seen in the above figures, the actual plateaus obtained in this study were tilted to some extent, and therefore we defined $G_{\mathrm{N}}^{0}$ as the $G^{\prime}$ value at $\omega a_{\mathrm{T}}$ where the loss tangent $\left(\tan \delta=G^{\prime \prime} / G^{\prime}\right)$ attained the minimum in the

144 rubbery plateau region (Horinaka et al. 2011; Horinaka et al. 2013). For example, $G_{N}^{0}$ for the HPC solution at $c=2.1 \times 10^{2} \mathrm{kgm}^{-3}$ is determined to be $1.9 \times 10^{4} \mathrm{~Pa}$ in Figure 5, which gives 
147 obtained in this manner are double-logarithmically plotted against $c$ in Figure 7. For each

148 polysaccharide, a straight line with a slope of -1 is drawn with the best fit method, because it

149 has been reported that a relation of $M_{\mathrm{e}} \propto c^{-1}$ holds for many concentrated solutions of polymers

150 (Doi et al. 1986; Masuda et al. 1972). It is seen that data points in the figure are fitted well by

151 the line, indicating that the $M_{\mathrm{e}} \propto c^{-1}$ relation also holds for the $\mathrm{C}, \mathrm{MC}$, and HPC solutions

examined in this study. Hence, $M_{\mathrm{e}, \text { melt }}$ for the cellulosic polysaccharides can be estimated to be $M_{\mathrm{e}}$ at $c=1.0 \times 10^{3} \mathrm{kgm}^{-3}$ of the fitted line in Figure 7 as we assume the density of the

154 polysaccharides to be $1.0 \times 10^{3} \mathrm{kgm}^{-3}$. The obtained values of $M_{\mathrm{e} \text {,melt }}$ are $2.9 \times 10^{3}, 2.5 \times 10^{3}$, and $6.2 \times 10^{3}$ for C, MC, and HPC, respectively. Here, it is noted that $M_{\mathrm{e}, \text { melt }}$ for C estimated in the current study is consistent within the experimental error with that for $\mathrm{C}$ obtained in our previous study where another ionic liquid 1-butyl-3-methylimidazolium chloride has been used as the solvent (Horinaka et al. 2011; Horinaka et al. 2012). The value of $M_{\mathrm{e}, \text { melt }}$ for MC obtained in this study is slightly smaller than that for C. Considering the experimental error, this difference might be negligible. On the other hand, $M_{\mathrm{e}, \text { melt }}$ for HPC is significantly larger. These results suggest that the effects of side groups on $M_{\mathrm{e}, \text { melt }}$ are apparent but not monotonous against $M_{\text {unit }}$ It has been reported earlier that there is an irregular tendency for the effect of $M_{\text {unit }}$ on a chain stiffness parameter of cellulosic polysaccharides in dilute solutions, although direct comparison with our result is impossible (Brown et al. 1964). It should be noted that 
aggregation behavior of the cellulosic polysaccharides does not account for the difference in

$M_{\mathrm{e} \text {,melt. }}$ It has been reported that side groups of cellulosic polysaccharides affects the solubility in

conventional solvents and extremely high substitution is necessary to obtain the molecularly

it is recognized that dissolution on the molecular level is achieved by forming hydrogen bonds

between the anions of the solvent and the hydroxyl protons of the solute, and therefore even

cellulose can be molecularly dissolved in ionic liquids, as demonstrated by a light scattering and 5 also indicate that there are no aggregates of the polysaccharides in all the solutions examined in this study; if the aggregates exist in the solution, another plateau (or at least shoulder), the so-called second plateau, should appear in the flow region before reaching the terminal behavior. Maeda et al. have explained the dynamic viscoelasticity data for an ionic liquid solution of cellulose over a wide range of frequency from the flow to the glassy zone successfully without taking the aggregated state of cellulose into consideration (Maeda, Inoue, Sato 2013). Now, we consider $N_{\text {unit }}$ instead of $M_{\mathrm{e}, \text { melt }}$. Since cellulosic polysaccharides have a common backbone structure of repeating glucose-ring units, the contour length between entanglement coupling points can be compared using $N_{\text {unit }}$ In other words, $N_{\text {unit }}$ represents the 
$M_{\mathrm{e}, \mathrm{melt}}$ and $M_{\text {unit }}$ are 18,13 , and 16 for C, MC, and HPC, respectively. The values of $N_{\text {unit }}$ come

\section{Conclusions}

187 The effects of side groups on entanglement network of cellulosic polysaccharide were examined in terms of the rheological properties $M_{\mathrm{e}, \mathrm{melt}}$ and $N_{\text {unit }}$. Dynamic viscoelasticity measurements for the concentrated solutions of C, MC, and HPC in BmimAc provided $M_{\mathrm{e} \text {,melt }}$ of $2.9 \times 10^{3}, 2.5 \times$ although the dependence appeared rather complicated. On the other hand, the effects of side groups were very small regarding $N_{\text {unit }}$.

References

Brown W, Henry D, Ohman J (1963) Studies on cellulose derivatives part II. The influence of solvent and temperature on the configuration and hydrodynamic behavior of hydroxyethyl cellulose in dilute solution. Makromol Chem 64: 49-67

Brown W, Henry D (1964) Studies on cellulose derivatives part III. Unperturbed dimensions of 
201 Burchard W (2003) Solubility and solution structure of cellulose derivatives. Cellulose 10:213-225.

Chen X, Zhang Y, Cheng L, Wang H (2009) Rheology of concentrated cellulose solutions in

Chen X, Zhang Y, Ke F, Zhou J, Wang H, Liang D (2011) Solubility of neutral and charged

Doi M, Edwards SF (1986) The theory of polymer dynamics. Clarendon Oxford

Flory PJ (1966) Treatment of the effect of excluded volume and deduction of unperturbed dimensions of polymer chains. Configurational parameters for cellulose derivatives.

Gericke M et al. (2009) Rheological properties of cellulose/ionic liquid solutions: from dilute to concentrated states. Biomacromolecules 10: 1188-1194.

Haward SJ et al. (2012) Shear and Extensional Rheology of Cellulose/Ionic Liquid Solutions.

Horinaka J, Yasuda R, Takigawa T (2011) Entanglement properties of cellulose and amylose in 
219 for linear D-glucans. Colloid Polym Sci 290: 1793-1797

220 Horinaka J, Urabayashi Y, Takigawa T, Ohmae M (2013) Entanglement network of chitin and chitosan in ionic liquid solutions. J Appl Polym Sci 130: 2439-2443

Kamide K, Miyazaki Y (1978) The partially free draining effect and unperturbed chain dimensions of cellulose, amylose, and their derivatives. Polym J 10: 409-431

Kamide K, Saito M (1987) Cellulose and cellulose derivatives: recent advances in physical chemistry. Adv Polym Sci 83: 1-56

Kosan B, Michels C, Meister F (2008) Dissolution and forming of cellulose with ionic liquids.

Meda A, Inoue T, Sato T (2013) Dynamic segment size of the cellulose chain in an ionic liquid.

Masuda T, Toda N, Aoto Y, Onogi S (1972) Viscoelastic properties of concentrated solutions of poly(methyl methacrylate) in diethyl phthalate. Polym J 3: 315-321

Onogi S, Masuda T, Kitagawa K (1970) Rheological properties of anionic polystyrenes. I.

Swatloski RP, Spear SK, Holbrey JD, Rogers RD (2002) Dissolution of cellose with ionic 
237 Cellulose 16: 9-17

238 
240 Figure Captions

241 Fig. 1 Master curves of $\omega$ dependence of $G^{\prime}$ and $G^{\prime \prime}$ for the $\mathrm{C}$ solutions at $T_{\mathrm{r}}=80{ }^{\circ} \mathrm{C}$. The curves are shifted upwards by $A$.

Fig. 2 Shift factor for the $\mathrm{C}$ solutions plotted against the reciprocal of $T$. All data points fall on a single line.

Fig. 3 Master curves of $\omega$ dependence of $G$ ' and $G$ ' for the MC solutions at $T_{\mathrm{r}}=80^{\circ} \mathrm{C}$. The curves are shifted upwards by $A$.

Fig. 4 Shift factor for the MC solutions plotted against the reciprocal of $T$. All data points fall on a single line.

Fig. 5 Master curves of $\omega$ dependence of $G$ ' and $G$ ' for the HPC solutions at $T_{\mathrm{r}}=80{ }^{\circ} \mathrm{C}$. The curves are shifted upwards by $A$.

Fig. 6 Shift factor for the HPC solutions plotted against the reciprocal of $T$. All data points fall on a single line.

Fig. 7 Double-logarithmic plot of $M_{\mathrm{e}}$ vs. $c$ for the cellulosic polysaccharides in solution. Each 
Figure 1

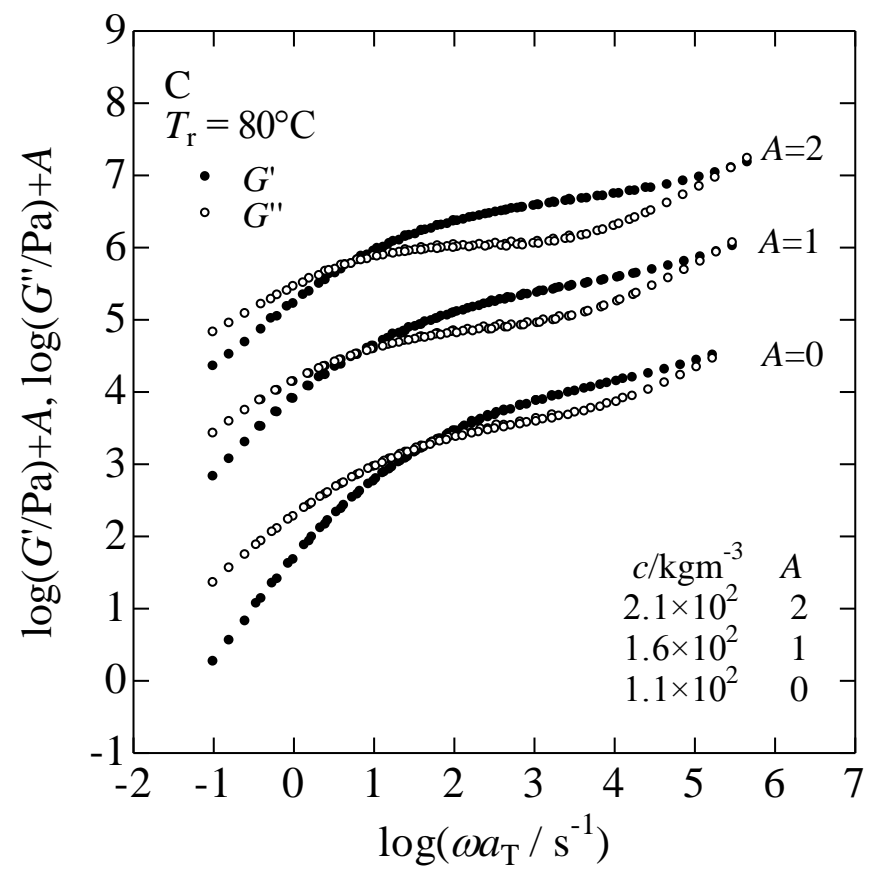


Figure 2

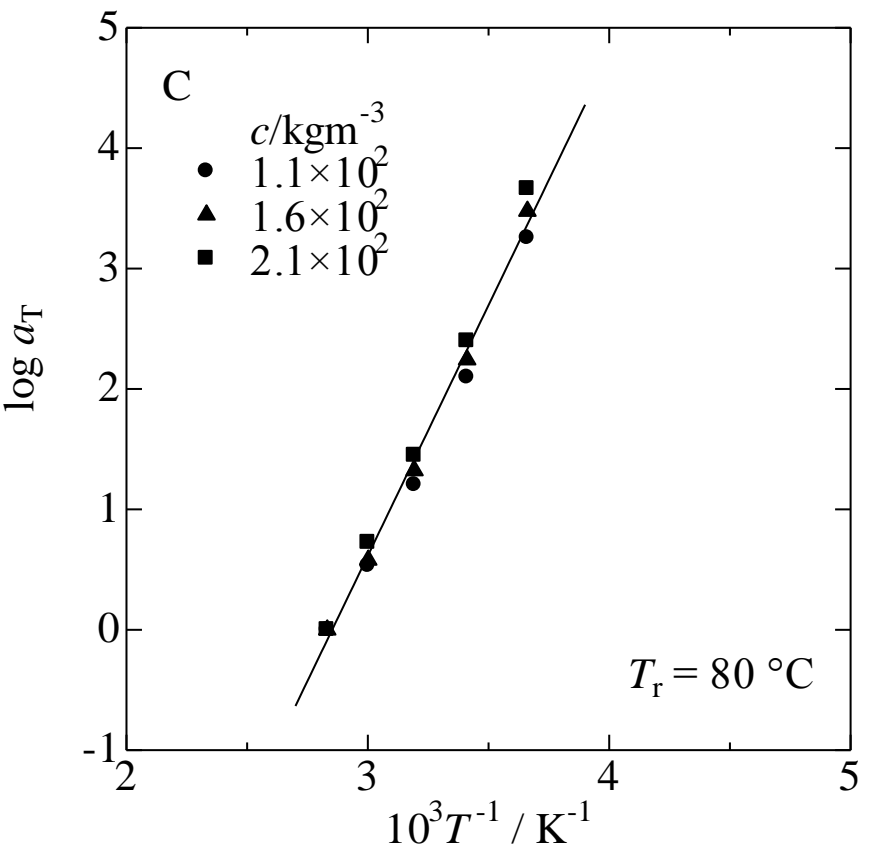


Figure 3

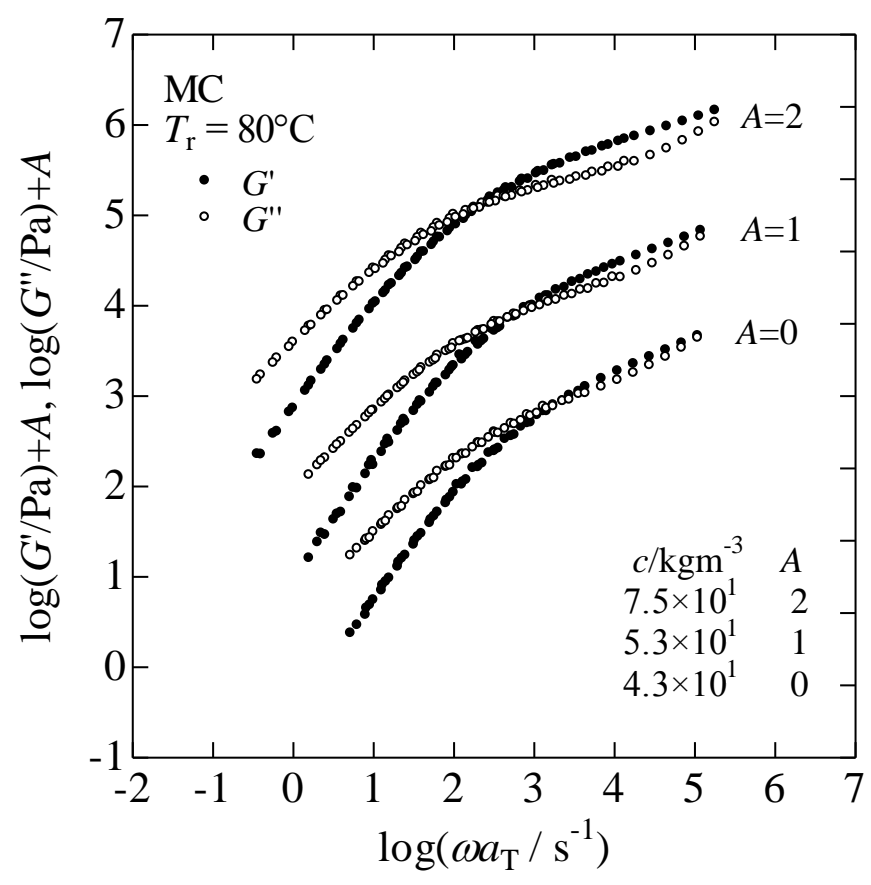


Figure 4

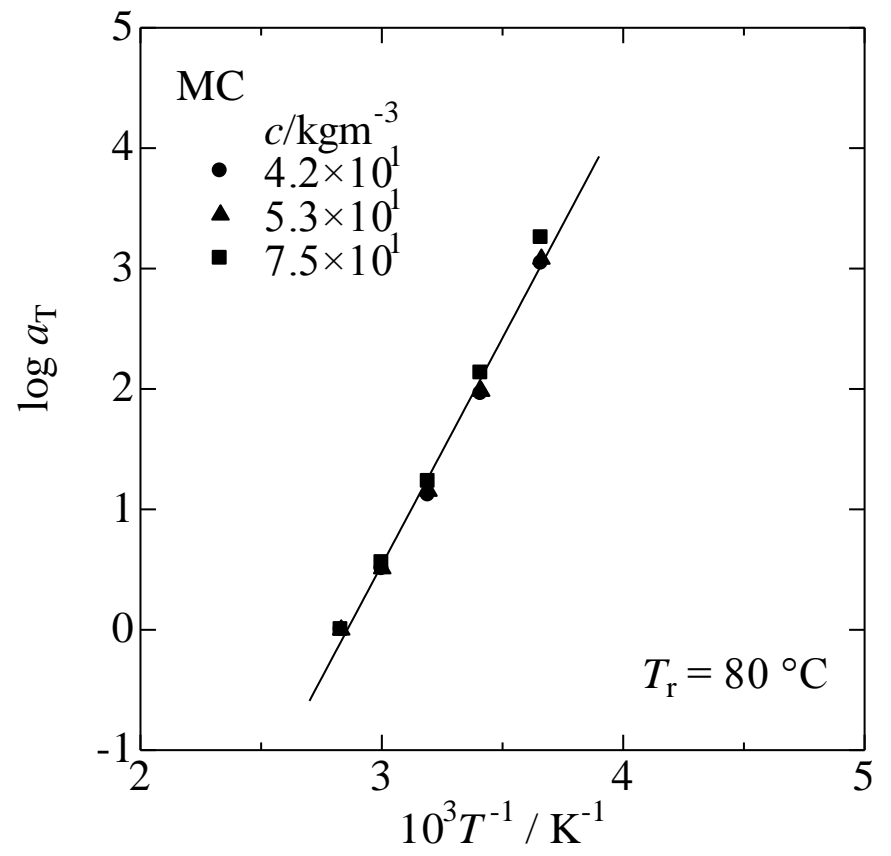


Figure 5

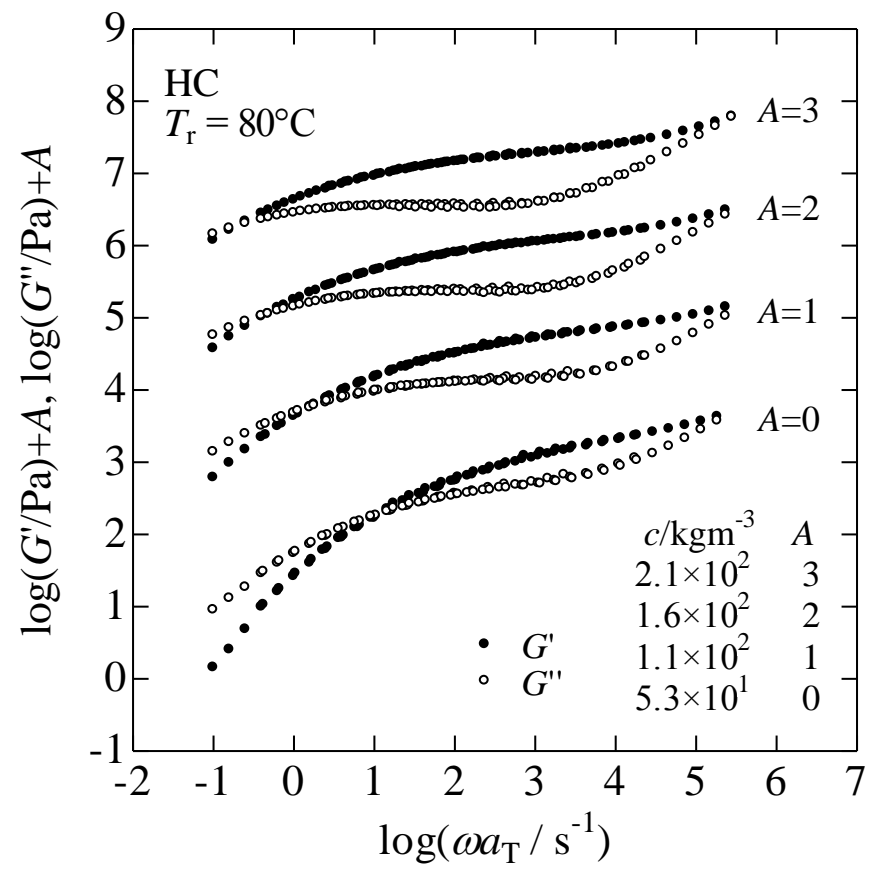


Figure 6

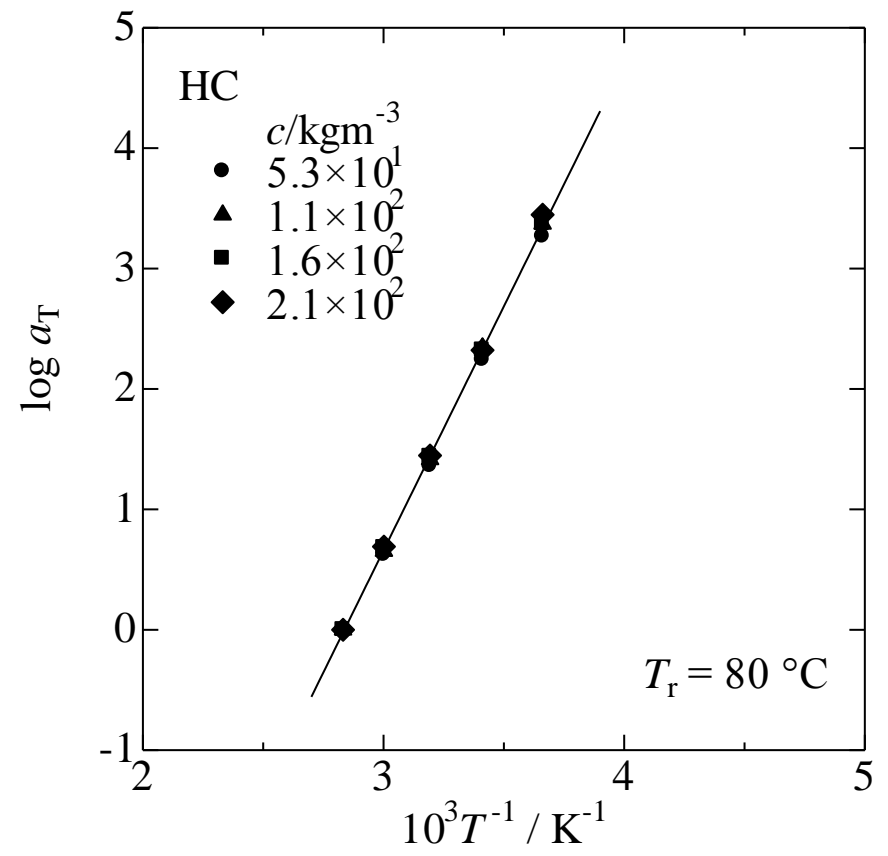


Figure 7

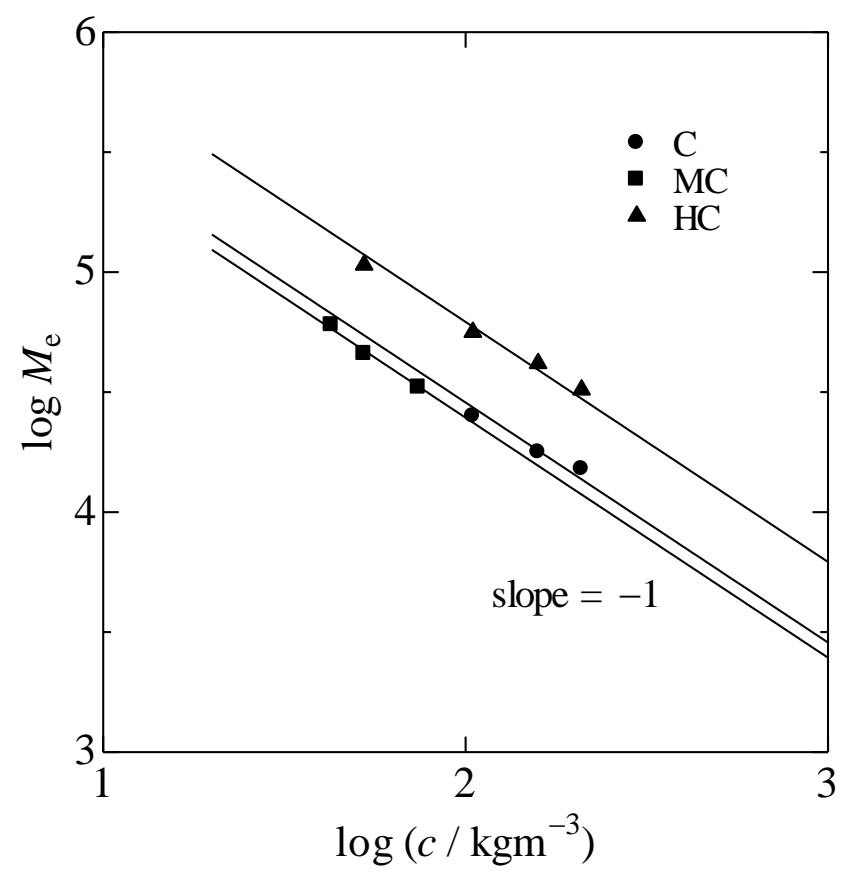

